\title{
Clues to Quasar Broad-Line Region Geometry and Kinematics
}

\section{Citation}

Vestergaard, M., B. J. Wilkes, and P. D. Barthel. 2000. “Clues to Quasar Broad-Line Region Geometry and Kinematics." The Astrophysical Journal 538 (2) (August 1): L103-L106. doi:10.1086/312805.

\section{Published Version}

doi:10.1086/312805

\section{Permanent link}

http://nrs.harvard.edu/urn-3:HUL.InstRepos:30248641

\section{Terms of Use}

This article was downloaded from Harvard University's DASH repository, and is made available under the terms and conditions applicable to Other Posted Material, as set forth at http:// nrs.harvard.edu/urn-3:HUL.InstRepos:dash.current.terms-of-use\#LAA

\section{Share Your Story}

The Harvard community has made this article openly available.

Please share how this access benefits you. Submit a story.

Accessibility 


\title{
CLUES TO QUASAR BROAD-LINE REGION GEOMETRY AND KINEMATICS ${ }^{1}$
}

\author{
M. VestergaArd, ${ }^{2,3,4}$ B. J. Wilkes, ${ }^{3}$ And P. D. Barthel ${ }^{5}$ \\ Received 2000 February 25; accepted 2000 June 1; published 2000 July 25
}

\begin{abstract}
We present evidence that the high-velocity C IV $\lambda 1549$ emission-line gas of radio-loud quasars may originate in a disklike configuration, in close proximity to the accretion disk often assumed to emit the low-ionization lines. For a sample of 36 radio-loud $z \approx 2$ quasars, we find the $20 \%-30 \%$ peak widths to show significant inverse correlations with the fractional radio core-flux density $R$, which is the radio axis inclination indicator. Highly inclined systems have broader line wings, consistent with a high-velocity field perpendicular to the radio axis. By contrast, the narrow line core shows no such relation with $R$, so the lowest velocity $\mathrm{C}$ IV-emitting gas has an inclination-independent velocity field. We propose that this low-velocity gas is located at higher disk altitudes than the high-velocity gas. A planar origin of the high-velocity $\mathrm{C}$ IV emission is consistent with the current results and with an accretion disk wind emitting the broad lines. A spherical distribution of randomly orbiting broadline clouds and a polar high-ionization outflow are ruled out.
\end{abstract}

Subject headings: galaxies: active — quasars: emission lines

\section{INTRODUCTION}

Little is currently known about the detailed geometry and structure of the broad-line region (BLR) in quasi-stellar objects (QSOs) due to the difficulty in spatially resolving the central regions, even in the most nearby active galactic nuclei (AGNs), to the limited sensitivity of photoionization processes to the detailed BLR geometry, and to the complex nature of reverberation transfer functions (Peterson 1997; Netzer \& Peterson 1997). For example, it is unclear whether the BLR gas has a spherical or disklike distribution and whether the BLR consists of discrete clouds or is part of a continuous gas distribution, such as an accretion disk wind (e.g., Boroson \& Green 1992, hereafter BG92; Murray \& Chiang 1997). An improved knowledge of the geometry and structure of the centrally emitting and absorbing gas regions and their relation to one another and to the continuum source(s) may help us to better understand the observed continuum-line and line-line correlations (e.g., BG92; Brotherton 1996; Wilkes et al. 1999; Wills et al. 1999) and may furthermore shed light on the interrelation of individual AGN subclasses.

The best constraints on the geometry and structure have so far been obtained from reverberation mapping (see the excellent reviews by Peterson 1993 and Netzer \& Peterson 1997) of lowluminosity AGNs showing that (1) the BLR is rather compact (the outer radius $R_{\text {outer }}$ is smaller than $0.3 \mathrm{pc} \approx 1 \mu \mathrm{as}$ ) but is geometrically thick, with $R_{\text {outer }} \gtrsim 10 R_{\text {inner }},(2)$ a certain degree of ionization stratification is present in the BLR gas, and (3) the BLR size and the (bolometric) luminosity of the active nucleus are correlated (e.g., Kaspi et al. 2000). Some clues to the relative position of the continuum source and the scattering,

\footnotetext{
${ }^{1}$ Observations reported in this Letter were obtained at the Multiple Mirror Telescope (MMT) Observatory, a facility operated jointly by the University of Arizona and the Smithsonian Institution.

${ }^{2}$ Columbus Fellow; present address: Department of Astronomy, The Ohio State University, 140 West 18th Avenue, Columbus, OH 43210-1173; vester@astronomy.ohio-state.edu.

${ }^{3}$ Harvard-Smithsonian Center for Astrophysics, 60 Garden Street, MS-4, Cambridge, MA 02138; bwilkes@cfa.harvard.edu.

${ }^{4}$ Niels Bohr Institute for Astronomy, Physics and Geophysics, Copenhagen University Observatory, Juliane Maries Vej 30, DK-2100 Copenhagen Ø, Denmark.

${ }^{5}$ Kapteyn Astronomical Institute, P.O. Box 800, NL-9700 AV Groningen, the Netherlands; pdb@astro.rug.nl.
}

emitting, and absorbing components, respectively, can be obtained from polarization studies of broad absorption line QSOs (e.g., Ogle 1997) that argue that they are highly inclined "radioquiet" QSOs. Additional and independent clues to the BLR gas kinematics may be obtained by studying the widths of the broad emission lines themselves. The gravitational potential of the central engine is thought to dominate the kinematics of the BLR gas, and the line width (FWHM) is thought to estimate the gravitational potential at the location of the gas (e.g., Peterson \& Wandel 1999). If the motion of the BLR gas has a preferred direction, as opposed to disorganized virial motions around the central black hole, we would expect the projection of this velocity field on our sight line to vary as the source axis inclination varies in the AGN population.

For a sample of broad-line radio sources, Wills \& Browne (1986) found $\mathrm{FWHM}(\mathrm{H} \beta)$ to relate to the radio core fraction, $\log R$, which is an estimator of the radio axis inclination to our line of sight, such that face-on sources lack very broad lines. They conclude that $\mathrm{H} \beta$ is emitted by a disk configuration perpendicular to the radio axis, which offers support to the twocomponent model for the high- and low-ionization lines (Collin-Souffrin et al. 1988). The low-ionization, very high density environment of the accretion disk fits the requirements of the physical conditions in the Balmer line-emitting gas. The low level of long-term variability in the broad $\mathrm{H} \beta$ wings (e.g., Maoz et al. 1994; Peterson et al. 2000) seems to support the disk interpretation (e.g., Corbin \& Smith 2000; but see Brotherton 1996 for counter arguments). Also, Zheng (1992) and Corbin (1997) model the high-velocity BLR emission and find consistencies with Keplerian motion in low- $z$ sources. CollinSouffrin et al. suggested a polar outflow origin for the highionization lines (HILs), thereby explaining their observed peak blueshift relative to the low-ionization lines (LILs; e.g., Gaskell 1982; Wilkes 1984; Tytler \& Fan 1992). However, in the absence of a definitive model, a spherical distribution of dense, discrete HIL-emitting clouds is often adopted in BLR studies to explain trends such as the inverse correlation between [O III] $\lambda 5007$ and the Fe II emission (BG92).

In order to explore these possible models, we studied the inclination dependence of the widths of $\mathrm{C}$ IV $\lambda 1549$, representative of the HILs, relative to those reported for $\mathrm{H} \beta$ in a sample of radio-loud quasars (RLQs). We find interesting constraints 
TABLE 1

Subsample Match Statistics

\begin{tabular}{lcccccc}
\hline \hline & & \multicolumn{4}{c}{$\log L_{\text {ext }}(5 \mathrm{GHz})$} \\
\cline { 3 - 6 } SAMPLE & Number & Mean & Median & Standard Error & Range & $M_{V}$ RANGE \\
\hline LDQ $\ldots \ldots$ & 26 & 27.75 & 27.75 & 26.84 & $26.89-28.13$ & -26.5 to -28.9 \\
CDQ $\ldots \ldots$ & 11 & 27.78 & 27.82 & 27.12 & $27.10-28.11$ & -27.0 to -29.2 \\
\hline
\end{tabular}

that are applicable to the HIL geometry, which we present below. This project is part of an extended study of the ultraviolet (UV) broad emission lines in moderate- $z$ QSOs with a range of radio power.

\section{DATA ANALYSIS}

The details of the sample and the optical data of the extended study are described by M. Vestergaard et al. (2000, in preparation, hereafter Paper I). We summarize the main points below. The radio data are described by Barthel et al. (1988), Lonsdale, Barthel, \& Miley (1993), and Barthel, Vestergaard, \& Lonsdale (2000). The current sample consists of 25 lobe-dominated quasars (LDQs; $R_{5}<0.5 ; R_{5}$ is defined below) and 11 coredominated quasars (CDQs; $\left.R_{5} \geq 0.5\right)$ chosen from the larger sample of $z \approx 2$ RLQs that were originally selected from Barthel, Tytler, \& Thomson (1990) and the 3C and 4C catalogs. To ensure that we study the inclination dependence in the line widths and not, say, a dependence on intrinsic source brightness, the CDQs and LDQs were selected such that their distributions of extended $5 \mathrm{GHz}$ radio luminosity $L_{\text {ext }}$ are similar. A Kolmogorov-Smirnov test confirms this to a confidence level (CL) of $97.7 \%$. The $L_{\text {ext }}$ ranges and the mean and median values also match to within the standard error, while the $M_{V}$ ranges match to within the expected uncertainty of $\sim 0.5 \mathrm{mag}$ (Table 1). Three of the LDQs are compact $(\leq 15 \mathrm{kpc})$, but their $R_{5}$-values could be measured from our high-resolution radio images. Highly variable RLQs are excluded. For the RLQs not presented by Barthel et al. (1990), we obtained MMT Blue Channel spectra during 1994 October-1996 November. The RLQs were observed at air masses below 1.4 and with a 1" slit placed at the parallactic angle. The Barthel et al. data were

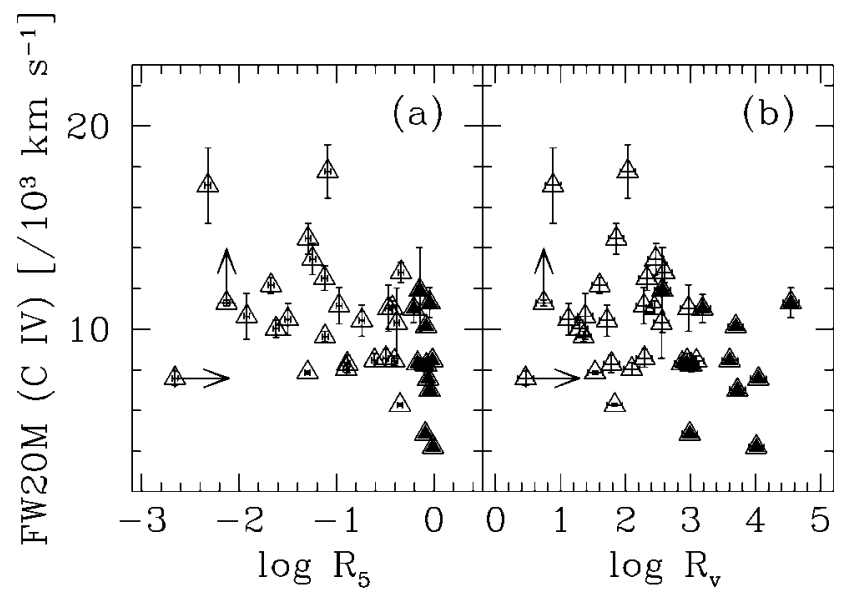

FIG. 1.-FW20M relations with (a) $\log R_{5}$ and $(b) \log R_{V}$. The CDQs are denoted by filled triangles. Spearman rank correlation tests show confidence levels of $(a) 99.7 \%$ and $(b) 96 \%$. The lower confidence level for $\log R_{V}$ is likely due to its relation being a zone of avoidance of very broad lines in CDQs and not a clear inverse correlation. not flux-calibrated well, and so they were recalibrated using short MMT exposures.

Spurious noise spikes and narrow absorption lines superposed on the emission lines were corrected by generating smooth fits to the profiles. The multiple Gaussian fitting (Laor et al. 1994; Paper I) that was applied to the C IV-He II line complex also allows a deblending of the line emission contributions, especially in the red wing of C IV, which in a few objects shows signs of weak Fe II emission. This is not expected to affect the current results adversely. The limitations imposed by the Fe II emission will be discussed in Paper I. The profile parameters were measured on the smooth $\mathrm{C}$ IV fits to ensure that they were measured consistently, objectively, and with minimum uncertainties. The full line widths at fractional profile heights Y $(=10 \%, 20 \%, \ldots, 90 \%)$ were determined (FWYM) along with the interpercentile velocity widths IPV(Y\%) (Whittle 1985). Reliable error estimates of each line parameter, based on continuum and flux uncertainties, were determined in a similar fashion as outlined by Whittle (1985; see Paper I for details).

The radio core fraction is defined here as $R_{5}=S_{\text {core }} / S_{\text {total }}$, where $S_{\nu} \sim \nu^{\alpha}$ are the observed fluxes at $5 \mathrm{GHz}$ measured from our VLA images; the spectral index $\alpha$ is defined as $\alpha_{5000 \mathrm{MHz}}^{408 \mathrm{~Hz}}$ (or $\alpha_{5000 \mathrm{MHz}}^{1400 \mathrm{Mz}}$ if the former is unavailable). A $K$-correction ${ }^{6}$ was applied to $R_{5}$ to permit a comparison in the rest frame: $R_{5}=S_{\text {core }} /\left[\left(S_{\text {core }}+S_{\text {ext }}\right)(1+z)^{\left(\alpha_{\text {ext }}-\alpha_{\text {core }}\right)}\right]$, where $\alpha_{\text {core }}=0$ and $\alpha_{\text {ext }}=\alpha_{\text {extended }}=-0.8 \quad$ (e.g., Kellerman \& Pauliny-Toth 1981). Our definition of $R_{5}$ is less sensitive to variations in the density of the environment of the radio source than, e.g., the $R$ defined as $S_{\text {core }} / S_{\text {ext }}$, especially for face-on objects, where $S_{\text {ext }}$ tends to be relatively weak. We also consider the inclination measure $\log R_{V}$ defined as the ratio of the $5 \mathrm{GHz}$ core flux density to the visual flux density with applied $K$-correction (Wills \& Brotherton 1995). It is also less sensitive to the density of the radio source environment.

\section{TRENDS WITH SOURCE INCLINATION}

FWHM(C IV) shows no relation with $\log R_{5}$ (the $\mathrm{CL}^{7}$ of the Spearman rank correlation test is $\sim 79 \%$ ). However, FW20M(C IV), which is representative of the width of the broad $\mathrm{C}$ IV base, shows a strong inverse correlation with $\log R_{5}$ at the $99.7 \%$ CL (Fig. 1a). Support for the reality of this effect comes from the similar relations for FW10M, FW30M, and FW40M (CLs: 97.5\%, 99.6\%, and 98.6\%, respectively). As an "area parameter," IPV(Y) is much less sensitive to the varying line-core strength which causes scatter in the FWYM measures (Paper I). The fact that IPV $(40 \%-70 \%)$ show an inverse correlation with $\log R_{5}$ at the $97 \%-98 \%$ CLs also supports the reality of the FW20M trend. The IPV(Y $<40 \%)$ is more sensitive to variations in the extreme wings than FWYM, which is evident from the larger scatter and errors. This does not

\footnotetext{
${ }^{6} H_{0}=50 \mathrm{~km} \mathrm{~s}^{-1} \mathrm{Mpc}^{-1}$ and $q_{0}=0$ are used throughout.
}

${ }^{7} \mathrm{CL}=1-P$, where $P$ is the probability of no correlation. 
jeopardize the above results for IPV(40\%-70\%) (Paper I). The $\log R_{V}$ measure shows similar relations as $\log R_{5}$, though with relatively lower significance. This is most likely due to the fact that a zone of avoidance is more evident in these relations (e.g., Fig. $1 b)$, though it is less apparent than that presented for $\mathrm{H} \beta$ by Wills \& Browne (1986). Brotherton (1996), however, finds clear anticorrelations for FW25M and FWHM of $\mathrm{H} \beta$ (but none for FW75M).

The C IV FW20-30M relations with core dominance must primarily be driven by inclination and not some other property for the following reasons: (1) The $L_{\text {ext }}$ distributions are similar for LDQs and CDQs. This is further confirmed by the at-themost weak FW20-30M- $L_{\text {ext }}$ relations (CL $\lesssim 95 \%$ ). (2) The lack of EW correlations with core dominance (CLs < 92\%) indicates that the (luminosity-normalized) line flux does not change with inclination. (3) The lack of FW70-90M relations with core dominance (CLs $<37 \%$ ) shows that there is no weakening of the narrow line core in LDQs which could yield an apparent but unreal FW20-30M inclination dependence. (4) No correlation with inclination is found for $M_{V}, L_{\text {ext }}$, and redshift.

The line width relations with inclination directly show that the high-velocity field projected along the line of sight increases with source inclination. The velocity field of BLR gas (in discrete clouds or a continuous distribution) in organized orbits around the source axis is expected to behave this way. The similar $\mathrm{H} \beta$ and $\mathrm{C}$ IV relations imply that, to first order, the kinematical structures of the two high-velocity line-emitting regions, and by inference those of HILs and LILs, are similar. Item 2 above also implies that the line and continuum fluxes are anisotropically emitted to about the same degree. Highly beamed sources are excluded, and the UV continuum is also not expected to be significantly beamed (e.g., Wills \& Brotherton 1995). Item 2 confirms this.

Although these results do not provide independent evidence for a disklike geometry, they are consistent with the highvelocity HIL gas being physically close to the accretion disk and with its predominant motion being in a plane parallel to the disk. However, the $\mathrm{C}$ IV emission cannot originate in the low-ionization accretion disk material itself while the $\mathrm{H} \beta$ emission may (e.g., Collin-Souffrin et al. 1988). We therefore propose that the high-velocity HIL gas is planar and that it lies in close proximity to the disk, with similar overall kinematics. In our ongoing work, we will readdress this FW20-30M inclination dependence issue with larger and optimally constructed $L_{\text {ext }}$ samples of LDQs and CDQs.

Our results argue against two possible BLR geometries: (a) the velocity field of a spherical distribution of randomly orbiting clouds is not expected to vary with inclination, and (b) a polar outflow of the HIL gas would show the opposite inclination dependence to that seen here for C IV. However, a planar HIL velocity field and geometry are consistent with a disk wind, in which the broad lines are emitted at the base of dense, optically thick winds evaporating off the accretion disk (Murray \& Chiang 1997, 1998). This model can successfully explain a number of observational details. Bottorff et al. (1997) and Königl \& Kartje (1994) present alternative disklike BLR models.

Item 3 above implies that the lowest velocity BLR gas has a random velocity field relative to inclination. It may not be physically associated with the high-velocity gas located close to the accretion disk. Low-density HIL gas of lower optical depth at higher disk altitudes, similar to the proposed intermediate-line region (ILR; e.g., Brotherton et al. 1994; cf. Sulentic \& Marziani 1999), fits this requirement; the altitude is low enough to maintain the high-ionization level. In the Murray \& Chiang (1997) disk-wind model, the narrow line core is emitted by the wind at much larger disk radii than the broad line base. This predicts similar inclination dependences for the high- and low-velocity fields in contrast to our results and so requires a modification of the model, possibly along the lines suggested above.

Brotherton (1996) finds very similar results for the inclination dependence of the high- and low-velocity $\mathrm{H} \beta$ emission in a sample of RLQs. The very broad line region/ILR picture, which he advocates, is not geometry specific but is fully consistent with the results and interpretations presented here. A possible common HIL and LIL high-velocity component may also be present in low- $z$ radio-loud AGNs (Marziani et al. 1996).

A disklike geometry of the HIL gas predicts that (1) other HILs (Ly $\alpha, \mathrm{N}$ v, Si IV + O IV], and He II) should show similar dependences on source inclination (such a study is underway), (2) the low-velocity dispersion $\left(\$ 2000 \mathrm{~km} \mathrm{~s}^{-1}\right)$ of the narrowest line core of the HILs should be independent of inclination, assuming this gas is located at relatively high disk altitudes, and (3) the $\mathrm{C}$ IV and $\mathrm{H} \beta$ profile parameters should behave similarly with varying source inclination, excluding the $\mathrm{C}$ IV narrow line core and the contribution to $\mathrm{H} \beta$ from the narrowline region. No detailed study exists of high-redshift QSOs; the FWHMs of $\mathrm{C}$ IV and $\mathrm{H} \beta$ correlate somewhat at low redshift (e.g., Wilkes et al. 1999). We are currently studying this issue for low-redshift QSOs, based in part on Hubble Space Telescope archival data.

\section{SUMMARY}

Despite years of study, little is known for certain about the geometry of the BLR of an AGN beyond its compact size ( $\lesssim$ light-weeks). The present results indicate that the highvelocity HIL gas is located in a disklike configuration close to the accretion disk, long thought to emit the LILs, and with similar kinematics. Our results argue against a spherical cloud distribution with random cloud velocities and a high-ionization polar outflow. If confirmed, the implications are intriguing; they may help to constrain interpretations of BLR data and to suggest new studies that may shed light on the detailed structure and kinematics of the AGN BLR.

We thank Drs. Paolo Marziani, Smita Mathur, and Brad Peterson for their comments on this Letter, Dr. Bev Wills for useful discussions on the $\log R_{V}$ measure, and Dr. Norm Murray on the disk-wind model. M. V. is very pleased to thank the Smithsonian Astrophysical Observatory for their hospitality and gratefully acknowledges financial support from the Columbus Fellowship at The Ohio State University, the Danish Natural Sciences Research Council (SNF-9300575), the Danish Research Academy (DFA-S930201), and a Research Assistantship at the Smithsonian Astrophysical Observatory through NASA grants NAGW4266, NAGW-3134, and NAG5-4089 to B. J. W. B. J. W. gratefully acknowledges financial support from NASA contract NAS8-39073 (Chandra X-ray Center). P. D. B. acknowledges contributions by Drs. David Tytler and George Miley in the early stages of this project and travel support by the Leids KerkhovenBosscha Fonds. 


\section{REFERENCES}

Barthel, P. D., Miley, G. K., Schilizzi, R. T., \& Lonsdale, C. J. 1988, A\&AS, 73, 515

Barthel, P. D., Tytler, D. R., \& Thomson, B. 1990, A\&AS, 82, 339

Barthel, P. D., Vestergaard, M., \& Lonsdale, C. J. 2000, A\&A, 354, 7

Boroson, T. A., \& Green, R. F. 1992, ApJS, 80, 109 (BG92)

Bottorff, M., Korista, K. T., Shlosman, I., \& Blandford, R. D. 1997, ApJ, 479, 200

Brotherton, M. S. 1996, ApJS, 102, 1

Brotherton, M. S., Wills, B. J., Francis, P. J., \& Steidel, C. C. 1994, ApJ, 430, 495

Collin-Souffrin, S., Dyson, J. E., McDowell, J. C., \& Perry, J. J. 1988, MNRAS, 232, 539

Corbin, M. R. 1997, ApJ, 485, 517

Corbin, M. R., \& Smith, P. S. 2000, ApJ, 532, 136

Gaskell, C. M. 1982, ApJ, 263, 79

Kaspi, S., Smith, P. S., Netzer, H., Maoz, D., Jannuzi, B. T., \& Giveon, U. 2000, ApJ, 533, 631

Kellermann, K. I., \& Pauliny-Toth, I. I. K. 1981, ARA\&A, 19, 373

Königl, A., \& Kartje, J. F. 1994, ApJ, 434, 446

Laor, A., Bahcall, J. N., Jannuzi, B. T., Schneider, D. P., Green, R. F., \& Hartig, G. F. 1994, ApJ, 420, 110

Lonsdale, C. J., Barthel, P. D., \& Miley, G. K. 1993, ApJS, 87, 63

Maoz, D., Smith, P. S., Jannuzi, B. T., Kaspi, S., \& Netzer, H. 1994, ApJ, 421,34
Marziani, P., Sulentic, J. W., Dultzin-Hacyan, D., Calvani, M., \& Moles, M. 1996, ApJS, 104, 37

Murray, N., \& Chiang, J. 1997, ApJ, 474, 91 1998, ApJ, 494, 125

Netzer, H., \& Peterson, B. M. 1997, in Astronomical Time Series, ed. D. Maoz, A. Sternberg, \& E. M. Leibowitz (Dordrecht: Kluwer), 85

Ogle, P. M. 1997, in ASP Conf. Ser. 128, Mass Ejection from Active Galactic Nuclei, ed. N. Arav, I. Shlosman, \& R. J. Weymann (San Francisco: ASP), 78

Peterson, B. M. 1993, PASP, 105, 247

1997, An Introduction to Active Galactic Nuclei (Cambridge: Cambridge Univ. Press)

Peterson, B. M., et al. 2000, ApJ, in press (astro-ph/0005433)

Peterson, B. M., \& Wandel, A. 1999, ApJ, 521, L95

Sulentic, J. W., \& Marziani, P. 1999, ApJ, 518, L9

Tytler, D., \& Fan, X.-M. 1992, ApJS, 79, 1

Whittle, M. 1985, MNRAS, 213, 1

Wilkes, B. J. 1984, MNRAS, 207, 73

Wilkes, B. J., Kuraszkiewicz, J., Green, P. J., Mathur, S., \& McDowell, J. C. 1999, ApJ, 513, 76

Wills, B. J., \& Brotherton, M. S. 1995, ApJ, 448, L81

Wills, B. J., \& Browne, I. W. A. 1986, ApJ, 302, 56

Wills, B. J., Laor, A., Brotherton, M. S., Wills, D., Wilkes, B. J., Ferland, G. J., \& Shang, Z. 1999, ApJ, 515, L53

Zheng, W. 1992, ApJ, 385, 127 